\title{
Dust-corrected surface photometry of M 31 from Spitzer far-infrared observations
}

\author{
E. Tempel ${ }^{1,2}$, A. Tamm ${ }^{1}$, and P. Tenjes ${ }^{1,2}$ \\ 1 Tartu Observatory, 61602 Tõravere, Estonia \\ e-mail: elmo@aai .ee \\ 2 Institute of Physics, Tartu University, Tähe 4, 51010 Tartu, Estonia \\ e-mail: [atamm; peeter.tenjes]@ut.ee
}

Received 26 March 2009 / Accepted 17 November 2009

\begin{abstract}
Aims. We create a model for recovering the intrinsic, absorption-corrected surface brightness distribution of a galaxy and apply the model to the nearby galaxy M31.

Methods. We constructed a galactic model as a superposition of axially symmetric stellar components and a dust disc to analyse the intrinsic absorption effects. Dust column density is assumed to be proportional to the far-infrared flux of the galaxy. Along each line of sight, the observed far-infrared spectral energy distribution was approximated with modified black body functions corresponding to dust components with different temperatures, thereby allowing us to determine the temperatures and relative column densities of the dust components. We applied the model to the nearby galaxy M31 using the Spitzer Space Telescope far-infrared observations for mapping dust distribution and temperature. A warm and a cold dust component were distinguished.

Results. The temperature of the warm dust in M 31 varies between 56 and $60 \mathrm{~K}$ and is highest in the spiral arms, while the temperature of the cold component is mostly $15-19 \mathrm{~K}$ and rises up to about $25 \mathrm{~K}$ at the centre of the galaxy. The intensity-weighted mean temperature of the dust decreases from $T \sim 32 \mathrm{~K}$ in the centre to $T \sim 20 \mathrm{~K}$ at $R \sim 7 \mathrm{kpc}$ and outwards. The scalelength of the dust disc is $\left(a_{0}\right)_{\text {dust }} \approx 1.8\left(a_{0}\right)_{\text {stars }}$. We also calculated the intrinsic $U, B, V, R, I$, and $L$ surface brightness distributions and the spatial luminosity distribution. The intrinsic dust extinction in the $V$-colour rises from $0.25^{\mathrm{m}}$ at the centre to $0.4^{\mathrm{m}}-0.5^{\mathrm{m}}$ at $R \simeq 6-13 \mathrm{kpc}$ and decreases smoothly thereafter. The calculated total extinction-corrected luminosity of M 31 is $L_{B}=(3.64 \pm 0.15) \times 10^{10} L_{\odot}$, corresponding to an absolute luminosity $M_{B}=-20.89 \pm 0.04 \mathrm{mag}$. Of the total $B$-luminosity, $20 \%(0.24 \mathrm{mag})$ is obscured from us by the dust inside M31. The intrinsic shape of the bulge is slightly prolate in our best-fit model.
\end{abstract}

Key words. galaxies: individual: Andromeda (M 31) - galaxies: photometry - galaxies: structure - dust, extinction - infrared: galaxies

\section{Introduction}

The importance of correcting astronomical observations for the impact of intervening dust has been known for a long time. Several direct and indirect methods have been developed for estimating light absorption and reddening by interstellar matter. However, because of the complexity of this process, the influence of dust is often ignored in studies of individual galaxies, as well as extensive statistical samples. Unfortunately, ignoring dust effects seriously undermines the reliability of the results, since it may lead to substantially wrong estimates of the shapes, luminosities, colours, and masses of the stellar components of galaxies (Driver et al. 2007; Padilla \& Strauss 2008; Graham \& Worley 2008).

Two significantly different approaches can be used to estimate intrinsic dust effects in external galaxies. Firstly, light extinction and reddening can be traced directly by comparing the properties of affected and unaffected fields within or behind a given galaxy. For example, in the presence of a partly occulted background galaxy, the optical thickness and extinction curve of dust inside the foreground galaxy can be measured directly (Holwerda et al. 2007). Unfortunately, suitable pairs of galaxies are very rare. Alternatively, extinction can be estimated by counting background galaxies seen through the foreground galaxy and by comparing their abundance to unobscured fields (González et al. 1998, 2003; Holwerda et al. 2005). The statistical nature and difficulties in detecting galaxies behind brighter regions (e.g. the bulge) of the foreground galaxy tend to reduce the applicability of this method.

The second approach exploits the law of conservation of energy - optical and ultra-violet radiation absorbed by dust has to be reradiated at the infrared (IR) and longer wavelengths. Data collected by the Infrared Astronomical Satellite (IRAS), the Infrared Space Observatory (ISO), the Spitzer Space Telescope, and the Submillimetre Common-User Bolometer Array (SCUBA) have significantly improved our understanding of the spatial distribution, temperature, and physical properties of interstellar dust grains, enabling the construction of radiation transfer models even down to the level of individual dust grains. In addition to revealing new facts about cosmic dust itself, these models enable restoration of the intrinsic photometric properties of galaxies for which direct methods of estimating light extinction are not applicable.

Since the thermal radiation from dust strongly depends on dust temperature, far-IR emission maps taken at several wavelengths are required for calculating dust density and for estimating the extinction of starlight. The first proof that dust temperature may significantly vary within a galaxy was noted from the IRAS data by Helou (1986) and Lonsdale \& Helou (1987). It was suggested that, in addition to the warmer dust associated with star-forming regions, a cooler and more diffuse dust component 
exists. Higher angular resolution far-IR maps of several galaxies by the ISO satellite indicated that the spatial distributions of the cold dust and the warm dust are different (Haas et al. 1998; Tuffs \& Gabriel 2003; Engelbracht et al. 2004; Hinz et al. 2004). It was also discovered that the cold dust disc is usually more extended than the stellar disc (Popescu \& Tuffs 2003).

By approximating the IRAS and SCUBA measurements of dust spectral energy distributions in 18 galaxies with twocomponent dust models, Vlahakis et al. (2005) found that cold dust has temperatures $17-24 \mathrm{~K}$ and warm dust $28-59 \mathrm{~K}$. By using the Spitzer, SCUBA and IRAS observations for a sample of 10 galaxies Willmer et al. (2009) derived the temperature range for the cold dust $18-24 \mathrm{~K}$ and for the warm dust $52-58 \mathrm{~K}$. The ratio of the cold dust mass to the warm dust mass in these galaxies is typically 100-2000. These values were derived by averaging across each of the galaxies; dust temperature may be considerably different in specific regions.

To determine the total mass of dust in galaxies, Li \& Draine (2001), Weingartner \& Draine (2001), and Draine \& Li (2007) proposed a model considering three kinds of dust grains and the radiation field, in which dust temperature variations from galaxy to galaxy are regulated by the effective intensity of the radiation field. This model has been successfully applied to 17 galaxies by Draine et al. (2007) and more recently to 57 galaxies by Muñoz-Mateos et al. (2009). Montalto et al. (2009) have used this model for studying M31.

More sophisticated analyses, yielding dust temperature as a function of position in a galaxy and thus enabling a detailed comparison to far-IR maps are carried out within radiative transfer models of stellar radiation in a dusty environment (e.g. Sauty et al. 1998; Silva et al. 1998; Bianchi et al. 2000; Popescu et al. 2000; Tuffs et al. 2004). Spatial resolution can reach 20 pc in these models (Bianchi 2008). However, this is still not enough to resolve individual dust clumps. The problem can be solved by separating the clumpy warm dust component from the diffuse component, as done by Silva et al. (1998) and Popescu et al. (2000).

In this paper, we create a three-dimensional galaxy model with axi-symmetric stellar populations. To estimate extinction and to restore the intrinsic luminosity and colour distributions of a galaxy, we add a dust disc to the model. We apply the model to the nearby spiral galaxy M31, using the Spitzer farIR maps (supplemented by the IRAS observations) to determine the dust distribution. The distribution of the dust temperature is estimated by approximating the far-IR spectral energy distribution with modified black body functions assuming the presence of a colder and a warmer dust component.

The following general parameters of M31 have been applied in our calculations: the inclination angle has been taken 77.5 (Walterbos \& Kennicutt 1988; de Vaucouleurs et al. 1991); the major axis position angle is $38.1^{\circ}$ (Walterbos \& Kennicutt 1987; Ferguson et al. 2002) and the distance to M31 is $785 \mathrm{kpc}$ (McConnachie et al. 2005), corresponding to the scale $1^{\prime}=228 \mathrm{pc}$.

\section{Construction of the model}

The physical properties of dust and the extinction of the stellar light of a galaxy are determined by an interplay between the spatial radiation field and the dust grains at each location within the galaxy. Calculations of the intrinsic extinction of a galaxy should thus be based on the spatial luminosity distribution of the galaxy and the spatial density distribution of the dust grains.

\subsection{Density distribution model for the stellar components}

In this subsection, we develop a sufficiently flexible model for describing the spatial distribution of the luminosity of a galaxy. A two-dimensional projection of the model can be compared to the observed surface brightness distribution and model parameters can be adjusted.

The model galaxy is given as a superposition of its individual stellar components. Each visible component is approximated by an ellipsoid of rotational symmetry with a constant axial ratio $q$; its spatial density distribution follows the law

$l(a)=l(0) \exp \left[-\left(\frac{a}{k a_{0}}\right)^{1 / N}\right]$

where $l(0)=h L /\left(4 \pi q a_{0}^{3}\right)$ is the central density and $L$ is the component luminosity; $a=\sqrt{r^{2}+z^{2} / q^{2}}$, where $r$ and $z$ are two cylindrical coordinates; $a_{0}$ is the harmonic mean radius that characterises the real extent of the component, independently of the structure parameter $N$. The coefficients $h$ and $k$ are normalising parameters, dependent on $N$. The definition of the normalising parameters $h$ and $k$ and their calculation is described in Appendix B of Tenjes et al. (1994). The luminosity density distribution (1) proposed by Einasto (1965) is similar to the Sérsic (1968) law for surface densities. Differences between the Sérsic law and the two-dimensional projection of Eq. (1) can be seen in Tamm \& Tenjes (2006).

Observations have demonstrated that the stellar disc can have a toroidal form in some galaxies, i.e. it does not continue to the centre. As suggested by Einasto (Einasto 1969; Einasto et al. 1980), the spatial density of a disc with a central hole can be expressed as a sum of two spheroidal mass distributions

$l_{\mathrm{disc}}(a)=l_{+}(a)+l_{-}(a)$

both of which can be approximated with the exponential law (1). Adopting a model disc with zero density at $r=0$ and nonnegative density at $l_{\mathrm{disc}}(a)>0$ gives the following relations between the parameters of the components $l_{+}$and $l_{-}: a_{0-}=\kappa a_{0+}$, $L_{-}=-\kappa^{2} L_{+}, q_{-}=q_{+} / \kappa$, where $\kappa<1$ is a parameter that determines the relative size of the hole in the centre of the disc. Structural parameters $N_{-}$and $N_{+}$should be identical.

Density distributions of all visible components are projected along the line of sight and their sum yields the surface brightness distribution of the model

$L(A)=2 \sum_{j} \frac{q_{j}}{Q_{j}} \int_{A}^{\infty} \frac{l_{j}(a) a \mathrm{~d} a}{\left(a^{2}-A^{2}\right)^{1 / 2}}$,

where $A$ is the major semi-axis of the equidensity ellipse of the projected light distribution and $Q_{j}$ are their apparent axial ratios $Q^{2}=\cos ^{2} i+q^{2} \sin ^{2} i$. The inclination angle between the plane of the galaxy and the plane of the sky is denoted by $i$. The summation index $j$ designates each visible component.

To approximate the luminosity distribution of a given galaxy, an arbitrary number of components can be used. Obviously, a larger number of components enables a better approximation of observations; however, to keep the model physically justified, additional information (e.g. observations of metallicity and kinematics distribution) should be used for distinguishing different components and for determining their number and extent. For the majority of nearby regular galaxies, three to five stellar components with density distribution (1) should be enough for describing the luminosity distribution. 


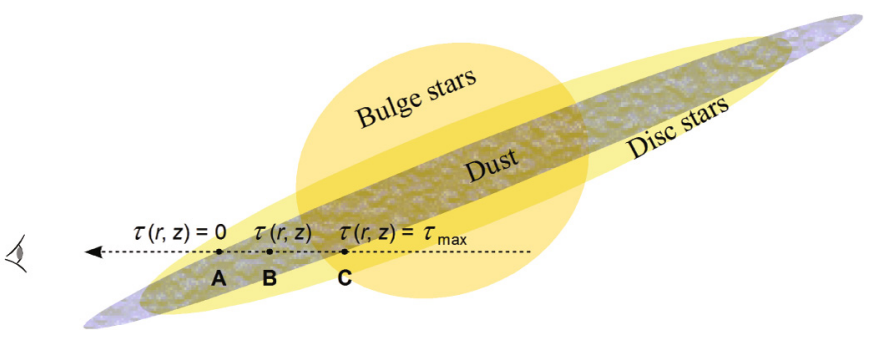

Fig. 1. Geometry of the dust disc model. The line-of-sight optical depth for three positions $\mathrm{A}-\mathrm{C}$ is indicated. The figure is illustrative only.

The model parameters of each component are determined by a least-squares approximation of the observed surface brightness distributions with the model profiles via Eq. (3). Uncertainties of the model can be estimated by using the partial second derivatives of the sum of the least-square differences (Bevington \& Robinson 2003).

\subsection{The dust disc component}

Let us now implant a dust disc component into our galaxy model, allowing us to calculate absorption along each line of sight through the galaxy.

Equation (3) will give the luminosity density along a line of sight, if no dust extinction is included. The intrinsic luminosity density for each component can be calculated from the equation

$L(X, Y)=\int_{X}^{\infty} \frac{\sum_{j=1}^{2}\left[l\left(r, z_{j}\right) \mathrm{e}^{-\tau\left(r, z_{j}\right)}\right]}{\sin i \sqrt{r^{2}-X^{2}}} r \mathrm{~d} r$,

$z_{1,2}=\frac{Y}{\sin i} \pm \frac{\sqrt{r^{2}-X^{2}}}{\tan i}$

where $l(r, z)$ denotes the spatial luminosity density (1) of the galaxy component, $L(X, Y)$ is the corresponding surface brightness distribution, $X$ and $Y$ are coordinates in the plane of the sky, and $\tau(r, z)$ is the optical depth along the line of sight (see Fig. 1).

Optical depth $\tau(r, z)$ is zero between the observer and the dust disc and $\tau(r, z)=\tau_{\max }$ behind the dust disc. $\tau_{\max }$ is the total optical depth along a given line of sight and thus a function of $(X, Y)$. Inside the dust disc, $\tau(r, z)$ is between 0 and $\tau_{\max }$.

The optical depth between the observer and each point inside the dust disc can be written as

$$
\tau(r, z)=\tau_{\max }(X, Y) \frac{\int_{\mathrm{A}}^{\mathrm{B}} n_{\mathrm{dust}}(s) \mathrm{d} s}{\int_{\mathrm{A}}^{\mathrm{C}} n_{\text {dust }}(s) \mathrm{d} s},
$$

where integration is done along the line of sight $s$ and $n_{\text {dust }}(s)$ is the spatial number density of the dust disc.

To gain a simple but nevertheless flexible model for the dust disc, we adopt the following form for $n_{\text {dust }}$ :

$n_{\text {dust }}(r, z)=f_{\text {dust }}(r) \cdot n(a)$,

where $n(a)$ is the exponential law (1) and $f_{\text {dust }}(r)$ describes deviations from the exponential law (1). The spatial number density of dust decreases exponentially in the $z$-direction according to Eq. (1). Multiplication by $f_{\text {dust }}(r)$ allows density behaviour to be more flexible along the $r$-direction.
Assuming that extinction is proportional to the dust column density and the latter is proportional to the far-IR flux, the parameters of $n(a)$, the shape of $f_{\text {dust }}(r)$ and subsequently the map of $\tau_{\max }(X, Y)$ can be derived. The relation between reddening and the far-IR flux can be expressed as

$E(B-V)=p D$,

where $D$ is a measure of the dust column density and $p$ is a calibration constant (Schlegel et al. 1998). Expressing $D$ as the $100 \mu \mathrm{m}$ flux of dust with a reference temperature $18.2 \mathrm{~K}$, Schlegel et al. (1998) have estimated $p=0.016 \pm 0.004$ by studying the colours of bright elliptical galaxies as seen through the Galactic dust.

To make Eq. (8) applicable for other galaxies, we need to take in account the difference between the $100 \mu \mathrm{m}$ flux of dust at the temperature $18.2 \mathrm{~K}$ and the actual flux of dust with a temperature $T$ at a wavelength $\lambda$. According to the modified Planck function

$D(X, Y)=F_{\lambda}(X, Y) \frac{\lambda^{\beta} B_{\lambda}(100 \mu \mathrm{m}, 18.2 \mathrm{~K})}{(100 \mu \mathrm{m})^{\beta} B_{\lambda}(\lambda, T(X, Y))}$,

where $F_{\lambda}(X, Y)$ is the measured far-IR flux map at a wavelength $\lambda$ and $T(X, Y)$ is the temperature map, $B_{\lambda}$ is the black body function and $\beta$ is the dust emissivity index. According to Vlahakis et al. (2005) we take $\beta=2.0$.

Using Eq. (8) and the relation $A_{V}=R \cdot E(B-V)$ and converting the derived extinction into optical depth, we reach a map of the total optical depth $\tau_{\max }(X, Y)$

$\tau_{\max , \lambda}(X, Y)=\frac{\ln (10)}{2.5} \frac{A_{\lambda}}{A_{V}} R p D(X, Y)$.

Xilouris et al. (1999) have found that in several galaxies, the extinction law agrees well with that of the Milky Way. This is also the case for M31 as indicated by the colours of its globular clusters (Barmby et al. 2000). We can thus use $p=0.016$ and $R=3.1$; the extinction ratio $A_{\lambda} / A_{V}$ can be determined from the Galactic extinction law.

Equation (10) gives the total extinction map in the plane of the sky. To calculate the actual intrinsic extinction, we also need the spatial density distribution of the dust disc $n_{\text {dust }}(r, z)$ for Eq. (6). We can compare the projection of the model space density distribution (calculated according to Eq. (3)) to the observed dust column density distribution (i.e. the far-IR maps) to determine the function $f_{\text {dust }}(r)$ and the parameters for $n(a)$. The observed column density of the dust disc is thereby converted into its spatial number density. More precisely, we can only determine the shape of the number density distribution of the dust disc; its absolute calibration $n(0)$ remains unknown. In the present case, the calibration constant is cancelled out in Eq. (6) and is not required for our model.

In the final step, the derived optical depth map $\tau_{\max , \lambda}(X, Y)$ and the function $n_{\text {dust }}(r, z)$ are inserted into Eq. (6) and the extinction-corrected surface brightness along each line of sight can be calculated from Eq. (4).

The relation between $\tau_{\max , \lambda}(X, Y)$ and reddening in Eq. (10) is calibrated according to the reddening of extragalactic sources by the dust of the Milky Way, thus including both extinction and scattering. On the other hand, the luminosities calculated from Eq. (4) include dust attenuation, but ignore the scattering of photons, leading to an overestimation of stellar emission in regions with high dust density. However, we consider the additional uncertainties caused by scattering to be lower than other potential error sources in $\tau$ determination. 
Table 1. Used photometrical data.

\begin{tabular}{|c|c|c|}
\hline Reference & $\begin{array}{l}\text { Filter } \\
\text { system }\end{array}$ & Remarks \\
\hline de Vaucouleurs (1958) & $B$ & ellipses \\
\hline Hoessel \& Melnick (1980) & $U B V$ & $\begin{array}{l}\text { major and } \\
\text { minor axis }\end{array}$ \\
\hline Hodge \& Kennicutt (1982) & $B$ & ellipses \\
\hline Kent (1987) & $R$ & ellipses \\
\hline Walterbos \& Kennicutt (1988) & $U B V R$ & ellipses \\
\hline Pritchet \& van den Bergh (1994) & $V$ & $\begin{array}{l}\text { star counts } \\
\text { (minor axis) }\end{array}$ \\
\hline Guhathakurta et al. (2005) & $V$ & $\begin{array}{l}\text { star counts } \\
\text { (minor axis) }\end{array}$ \\
\hline Irwin et al. (2005) & $V I$ & $\begin{array}{l}\text { ellipses and } \\
\text { star counts } \\
\text { (minor axis) }\end{array}$ \\
\hline Barmby et al. (2006) & $L$ & ellipses \\
\hline
\end{tabular}

Notes. "Ellipses" denote elliptically averaged surface brightness profiles.

\section{Applying the model to M 31}

\subsection{Optical data}

A large collection of surface brightness distribution measurements is available for modelling the stellar luminosity distribution in M31; see Tenjes et al. (1994) for references to earlier data. For the present work we have used the measurements of $U$, $B, V, R$, and $I$ distributions excluding some of the oldest, mainly photometrical data; however, for a more complete coverage of $B$ surface brightness distribution, we have also included the photoelectrical measurements by de Vaucouleurs (1958). As an example of nearly absorption-free observations, we have added the $3.6 \mu \mathrm{m}$ measurements by the Spitzer Space Telescope (Barmby et al. 2006) to our dataset, which roughly correspond to the Johnson-Cousins $L$ filter. Since we did not intend to model the nuclear part of M31, data at $r<0.3 \mathrm{kpc}$ were neglected. Table 1 presents references to the observations and the corresponding filter systems used in this paper. Some of the data are one-dimensional measurements along either or both of the axes of the galaxy, others are elliptically averaged contours of the galaxy. Measurements along the axes are useful for an accurate determination of the three-dimensional shape of the stellar populations, while ellipses provide the average surface brightness at each distance from the centre. Of the minor axis data, only those from the side closer to us (the southeast direction) have been used and are presented on figures throughout this paper. Different $R$ and $I$ colour system profiles were transferred into the Johnson-Cousins system, using the calibrations by Frei \& Gunn (1994). All measurements were corrected for extinction by dust of the Milky Way according to Schlegel et al. (1998).

\subsection{Infrared data}

Our analysis of the far-IR emission from M31 is based on the highest spatial resolution mappings currently available, the Spitzer Space Telescope MIPS camera imaging at 24, 70, and $160 \mu \mathrm{m}$ (Gordon et al. 2006), supplemented by the IRAS satellite detections at $100 \mu \mathrm{m}$. The only measurements available at longer wavelengths are those by the DIRBE instrument aboard the COBE satellite at up to $240 \mu \mathrm{m}$. Because of their very low angular resolution, the DIRBE observations were not suitable for this work.
The Spitzer images were downloaded using the Leopard toolkit developed at the Spitzer Science Center. An inspection of the pipeline-created (post-BCD) mosaic images revealed uneven background levels and the presence of a number of instrumental defects, making them unusable for our purposes. We started from the basic-calibrated data (BCD) and conducted the necessary corrections, stacking and mosaicing using the standard procedures within the IRAF and MOPEX software. The first three frames of each $24 \mu \mathrm{m}$ observing run, suffering from a shorter exposure time, were discarded. Depending on the fields, either a constant or a low order polynomial background was subtracted from the images. The IRAS imaging was retrieved from the NASA/IPAC Infrared Science Archive. The resolution of the IRAS data are only 90 arcsec/pixel; they were not used during the final approximation of the spectral energy distribution. The final images were sampled to 8 arcsec/pixel, which is the sampling of the $160 \mu \mathrm{m}$ observations; this also became the spatial resolution of our dust model.

Uncertainties were estimated considering three principal error sources: the characteristics of the telescope detectors, the precision of the absolute calibration and the uncertainty of the background level. An estimate for the response uncertainty of each detector pixel is indicated by the corresponding standard deviation maps; these were stacked and resampled by the MOPEX software during the mosaicing process. The conservative estimates of the uncertainties of the instrumental flux calibration of Spitzer were taken from Engelbracht et al. (2007), Gordon et al. (2007), and Stansberry et al. (2007): 4\%, 7\%, and 12\% for 24,70 , and $160 \mu \mathrm{m}$, respectively. Beichman et al. (1988) give $2 \%$ as the maximal variation in the IRAS absolute calibration. Possible background errors were estimated from deviations between the background flux density levels of neighbouring data stripes. Combining these three error sources, uncertainty maps were produced for each image, to be later used for calculating possible errors in our extinction analysis. In addition, the uncertainty of disentangling the contributions of M31 and the Milky Way from the far-IR maps has to be kept in mind. According to the IRAS maps, the sky brightness at $100 \mu \mathrm{m}$ around M 31 varies between 2 and $5 \mathrm{MJy} \mathrm{sr}^{-1}$ (Schlegel et al. 1998). This also gives an idea about a possible error resulting from wrong addressing of dust in the direction of M 31. We have not included this foreground uncertainty in our error estimates.

To probe the repeatability of our image processing and photometry, let us compare our estimate for the integral IR flux to some earlier measurements. The total sky-subtracted flux within the outermost ellipse fitted to the galaxy with the IRAF/STSDAS task ellipse is $119 \pm 7 \mathrm{Jy}, 1200 \pm 110 \mathrm{Jy}$, and $7800 \pm 1000 \mathrm{Jy}$ for the 24,70 , and $160 \mu \mathrm{m}$ images, respectively. These correspond rather well to the values $107 \pm 10 \mathrm{Jy}, 940 \pm 188 \mathrm{Jy}$, and $7900 \pm$ 1580 Jy measured by Gordon et al. (2006) inside a rectangular aperture $\left(2.75^{\circ} \times 0.75^{\circ}\right)$. The variations probably come from different methods used for background subtraction and from the aperture selection.

\subsection{Mapping dust temperature and optical depth}

Following Eqs. (9) and (10), we need a far-IR emission map and a map of the dust temperature. Since we are relying on the relation between reddening and the far-IR flux at $100 \mu$ m determined by Schlegel et al. (1998) for the Milky Way, the far-IR map has to correspond to the $100 \mu \mathrm{m}$ flux. Although the IRAS satellite has observed at this wavelength, we derived the necessary map by interpolating the Spitzer observations because of their higher spatial resolution. 


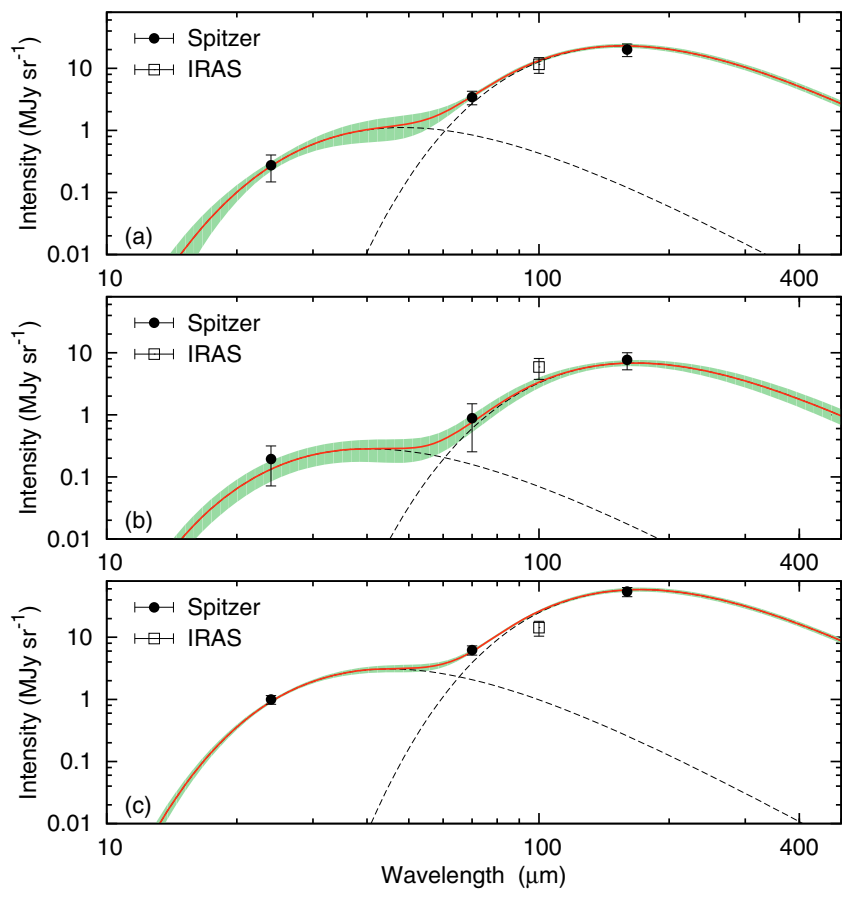

Fig. 2. Examples of approximations of the observed Spitzer (filled circles) and IRAS (empty squares) data with the modified black body functions at three random locations: a) in the bulge region; b) in an interarm region; c) in a spiral arm. In each panel, the black dashed lines represent the warm and the cold component and the red solid line is the sum of the two modified black body functions together with its uncertainty region.

Gordon et al. (2006) have shown that a two-component dust model gives a good fit to the far-IR spectral energy distribution of $\mathrm{M} 31$. The warmer $(T \approx 59 \mathrm{~K})$ dust component is about $10^{4}$ times less massive than the colder $(T \approx 17 \mathrm{~K})$ component, but it might still cause significant light extinction in specific regions, since it is considerably more clumpy and more concentrated in the galactic centre and in the spiral arms. Therefore, we have fitted the far-IR emission within each line of sight with two modified Planck functions, representing these two dominant temperature components.

To determine the intensity and temperature of both of the dust components, the modified Planck functions were fitted to the 4-bin spectral energy distribution observations (24, 70, 100, and $160 \mu \mathrm{m}$ ) in three stages for each line of sight (i.e. within each model pixel). The main contribution to the warm component comes from the star-forming regions. We assumed that the temperature of dust in these regions varies only slightly and fixed the temperature of the warm component at $T=59 \mathrm{~K}$ (Gordon et al. 2006) during the first fitting stage; the other parameters were derived using the Spitzer data. During the second stage, the IRAS data were added and the temperature of the warm dust was adjusted at lower spatial resolution. Finally, IRAS data were omitted again and the intensities of the dust components and the temperature of the cold dust were refined at higher spatial resolution. The uncertainties of the derived intensities and temperatures were estimated by using the Monte Carlo method, allowing the data points to vary randomly within their individual uncertainties. For each model pixel, 100 different sets of data points were created and approximated with the two-component model. Examples of the black body fits to the data points for three randomly selected lines of sight (i.e. for three model pixels) are given in Fig. 2. The final temperature maps and $100 \mu \mathrm{m}$
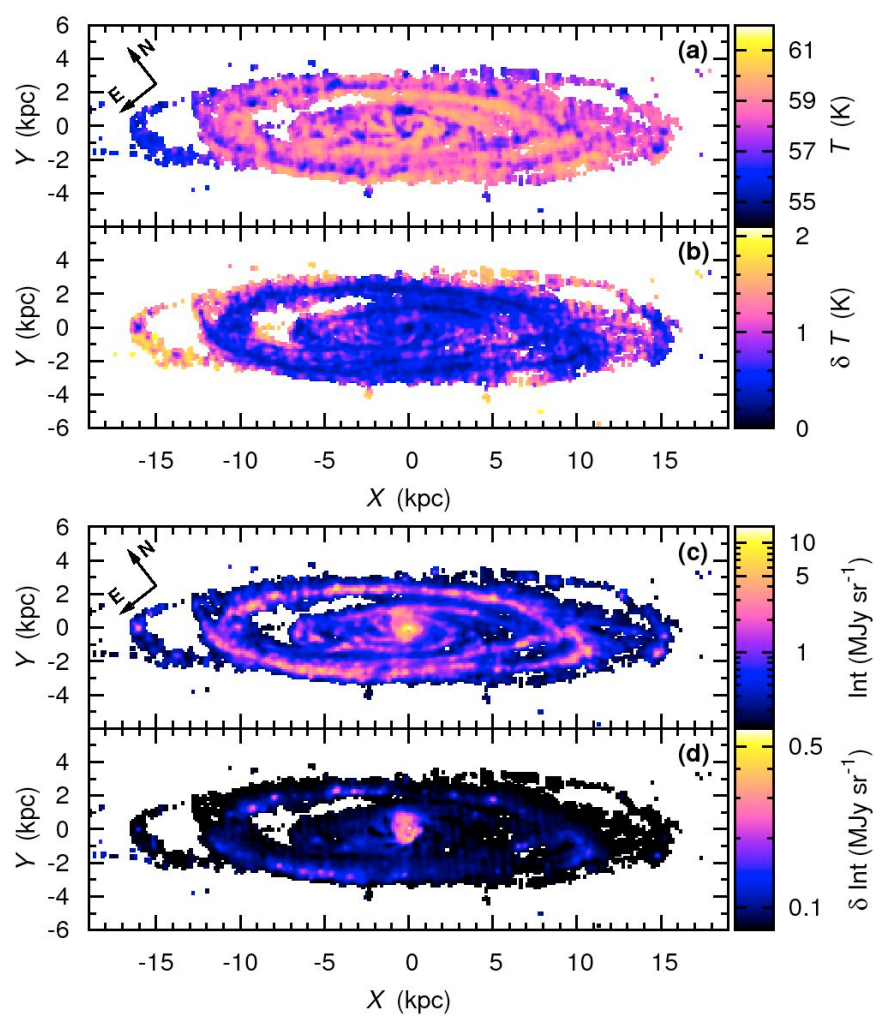

Fig. 3. Temperature and intensity maps of the warm dust component. In panels a) and b) the warm dust temperature and its $1 \sigma$-errors are given. In panels c) and d) the intensity of the warm component at $100 \mu \mathrm{m}$ and its $1 \sigma$-errors are given.

intensity maps of the two dust components are presented in Figs. 3 and 4 together with the corresponding $1 \sigma$-error maps.

Next, the total optical depth $\tau_{\max }$ (applicable to light sources behind the dust disc) was calculated for each model pixel according to Eqs. (9) and (10). Only the cold dust component was used for calculating the optical depths, thus the calculated opacities refer to the diffuse dust component. Since the mass of the warm dust component is very low (see Sect. 4), its contribution to the optical depth would be negligible. The derived $\tau_{\max , V}(X, Y)$ map was four-folded (see Fig. 5a) and approximated with an axially symmetric distribution (7) as described in Sect. 2.2 (see Fig. 5b). The residual map, indicating deviations between the axially symmetric model and the four-folded map is shown in Fig. 5d.

Approximating the far-IR maps with the axially symmetric distribution (7) does no provide an independent estimate for the vertical thickness of the dust disc component. We have used a rather conventional assumption that the mean ratio of the thickness of the stellar disc to the thickness of the dust disc is $1.8 \pm$ 0.6 , derived by modelling a sample of nearby edge-on galaxies by Xilouris et al. (1999). However, in some galaxies the dust disc appears thinner (Bianchi 2007), thus we have made comparative calculations also for the cases of a thinner and a thicker dust disc (see Sect. 5 for models with different dust disc thicknesses). The scalelength of the dust disc was determined during the approximation process of the far-IR emission maps. The structural parameters for the exponential approximation of the dust disc density distribution $n(a)$ are given in Table 2. The dust disc density profile (7) in the $r$-direction is given in Fig. 5c, also showing that without a correction function $f_{\text {dust }}(r)$, the simple exponential function $n(a)$ provides a very poor approximation. 

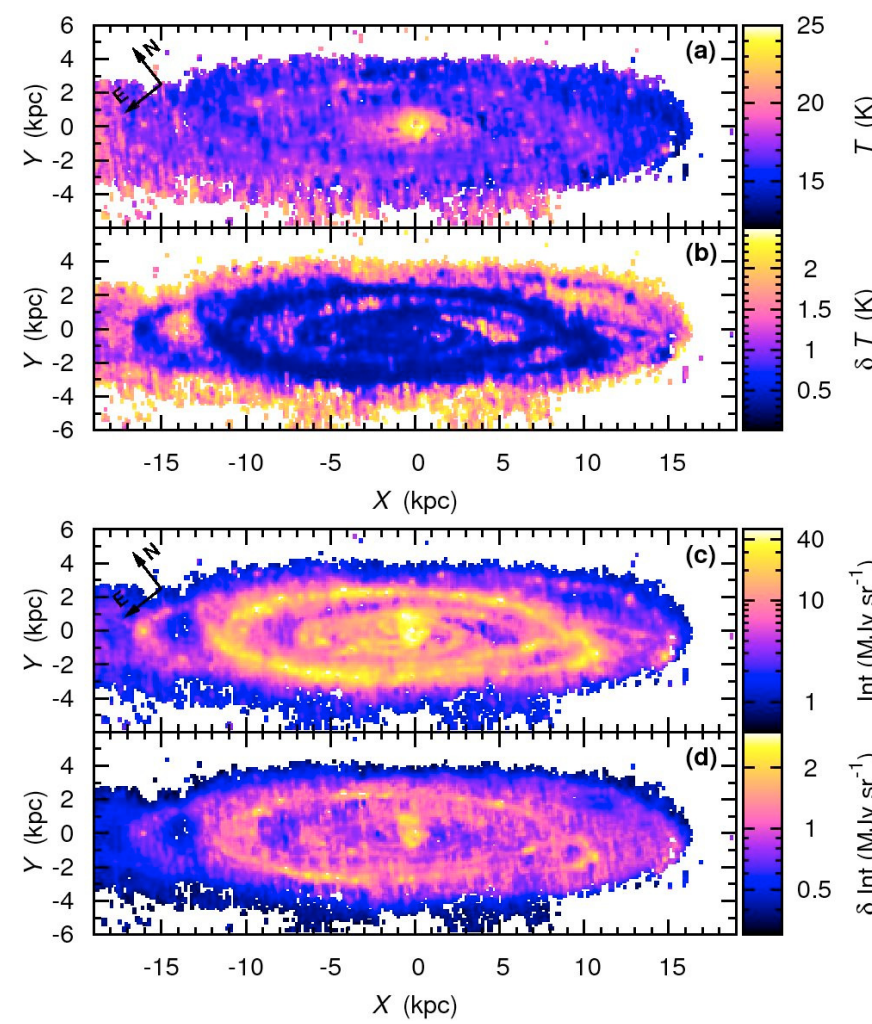

Fig. 4. Temperature and intensity maps of the cold dust component. In panels a) and b) the cold dust temperature and its $1 \sigma$-errors are given. In panels c) and d) the intensity of the cold component at $100 \mu \mathrm{m}$ and its $1 \sigma$-errors are given.

\subsection{Model fitting}

Prior to starting the actual fitting process, a reasonable number of different stellar components to be incorporated in the model was specified.

The two most dominant components of M31 are its bulge and disc, detectable even with an amateur telescope. In addition, recent studies suggest the presence of a dynamically warm but also slowly rotating stellar population of stars with moderate metallicity beginning at the outskirts of the bulge region. Here we designate this population as the halo; note that the same region has been referred to as the bulge (Kalirai et al. 2006), the extended disc (Ibata et al. 2005, 2007), the spheroid (Brown et al. 2007), and the outer halo (Durrell et al. 2001), depending on the viewpoint and the focus of each study. Furthermore, recent observational studies of distant fields in the direction of the minor axis have provided evidence for the existence of stellar populations far beyond the conventional bulge and halo regions (Irwin et al. 2005; Ibata et al. 2007). The outermost regions seem to be dominated by a faint, diffuse, very extended metal poor halo, possibly embedding much of the whole Local Group. Since it has almost negligible surface brightness and total luminosity, we do not model this population as a separate component.

Also, we made no attempt to include the Giant Stream or any other of the recently detected stellar streams in our model separately. Their contribution to the surface brightness, either measured along the axes of the galaxy or averaged along elliptical contours is insignificant.

Consequently, the fitting process was started with three stellar components: a bulge, a disc, and a halo. However, we soon realised that an additional component is necessary for a better description of the blue star-forming ring at about $10 \mathrm{kpc}$ from
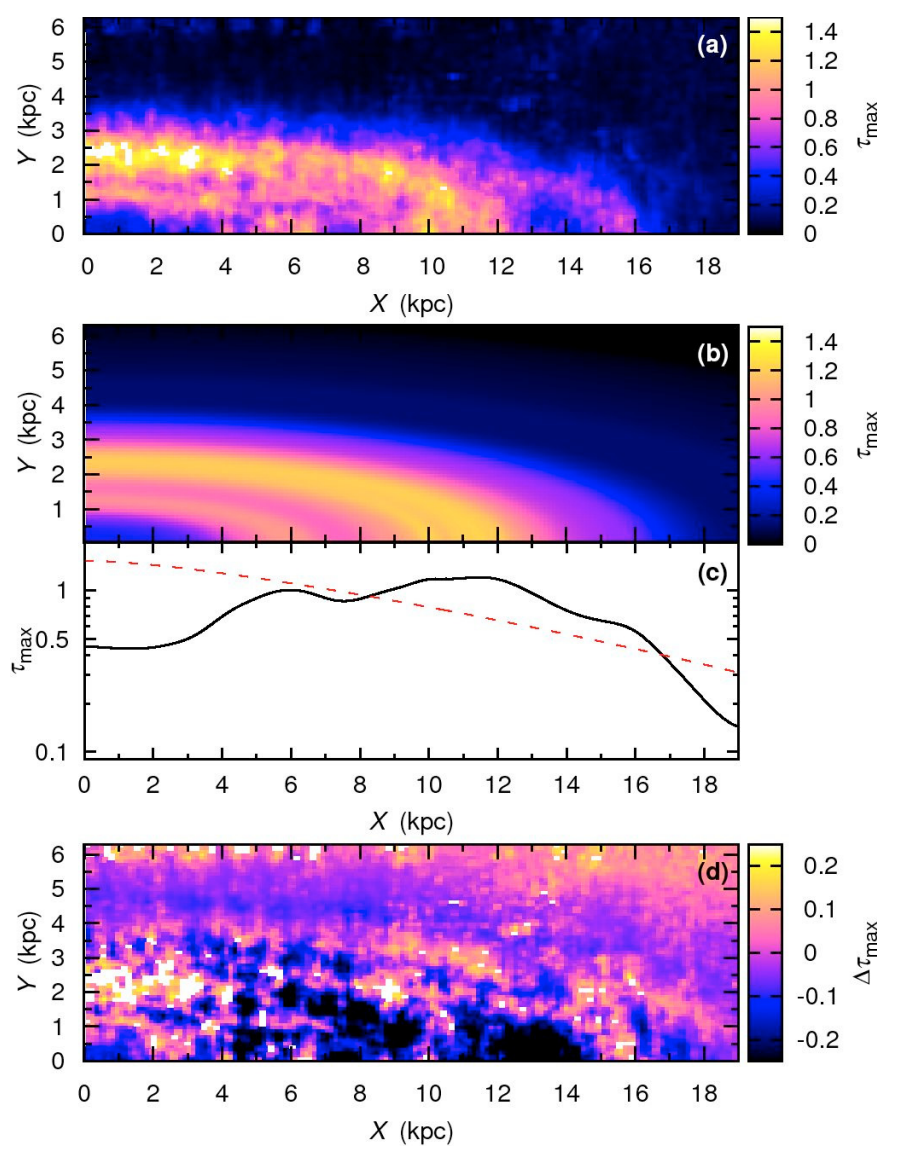

Fig. 5. Four-folded optical depth map of M31, derived from the cold dust component using Eq. (10). Panel a) - the observed optical depth; panel b) - the modelled optical depth along the line of sight; panel c) a cut from panel b) for $Y=0$ (black solid line); the red dashed line corresponds to the dust disc model with a simple exponential density distribution law (1); panel d) - the residual map of the observed and the modelled optical depth.

the centre, also coinciding with a peak in the distribution of gas clouds and other objects typical of regions of star formation (Einasto et al. 1980; Tenjes \& Haud 1991; Tenjes et al. 1994). The importance of considering clumpy young populations in models of the intrinsic dust extinction of galaxies is also stressed in radiative transfer models (e.g. Bianchi 2008). We inserted the young stellar population of M31 in our model as a disc with a central hole described by Eq. (2); we refer to this population as the "young disc" in the following.

The model parameters $q, a_{0}, L, N$, and $\kappa$ for each component were determined by a subsequent least-squares approximation process. First, we made a crude estimation of the population parameters. The purpose of this step is to avoid obviously non-physical parameters - relation (4) is non-linear and fitting a model to the observations is not a straightforward procedure. Next, a mathematically correct solution was found for each component. Details of the least-squares approximation and the general modelling procedure have been described by Einasto \& Haud (1989), Tenjes et al. (1994), and Tenjes et al. (1998). Uncertainties of the model due to observational uncertainties and the coupling of parameters were estimated using the partial second derivatives of the sum of the least-square differences (Bevington \& Robinson 2003). For each parameter, the calculated errors only reflect the goodness of the given fit; they do not represent the uniqueness of the model. Models with 
Table 2. Calculated parameters of the photometrical model.

\begin{tabular}{|c|c|c|c|c|c|c|}
\hline Population & $\begin{array}{l}a_{0} \\
(\mathrm{kpc})\end{array}$ & $q$ & $N$ & $\kappa$ & $k$ & $h$ \\
\hline Bulge & $0.50 \pm 0.01$ & $1.06 \pm 0.03$ & $3.46 \pm 0.11$ & & $7.2086 \times 10^{-4}$ & 896.897 \\
\hline Disc & $6.7 \pm 0.2$ & $0.08 \pm 0.01$ & $1.90 \pm 0.13$ & & $6.4723 \times 10^{-2}$ & 26.7655 \\
\hline Young $\operatorname{disc}^{a}$ & $9.5 \pm 0.2$ & $0.02 \pm 0.003$ & $0.35 \pm 0.02$ & $0.67 \pm 0.02$ & 1.3334 & 1.23802 \\
\hline Halo & $6.5 \pm 0.4$ & $0.50 \pm 0.02$ & $5.0 \pm 0.2$ & & $4.1625 \times 10^{-6}$ & 31809.6 \\
\hline Dust disc & 17.0 & 0.014 & 1.0 & & 0.5000 & 4.00000 \\
\hline Population & $\begin{array}{ll}L_{U} & \\
\left(10^{10} L_{\odot}\right)\end{array}$ & $\begin{array}{l}L_{B} \\
\left(10^{10} L_{\odot}\right)\end{array}$ & $\begin{array}{ll}L_{V} & \\
\left(10^{10} L_{\odot}\right)\end{array}$ & $\begin{array}{l}L_{R} \\
\left(10^{10} L_{\odot}\right)\end{array}$ & $\begin{array}{l}L_{I} \\
\left(10^{10} L_{\odot}\right)\end{array}$ & $\begin{array}{l}L_{L} \\
\left(10^{10} L_{\odot}\right)\end{array}$ \\
\hline Bulge & $0.63 \pm 0.07$ & $0.82 \pm 0.04$ & $(0.96 \pm 0.05)$ & $(0.93 \pm 0.04)$ & $0.83 \pm 0.09$ & $4.08 \pm 0.22$ \\
\hline Disc & $0.61 \pm 0.07$ & $0.90 \pm 0.06$ & $1.36 \pm 0.07$ & $1.47 \pm 0.13$ & $(1.88 \pm 0.27)$ & $5.40 \pm 0.52$ \\
\hline Young $\operatorname{disc}^{b}$ & $0.98 \pm 0.11$ & $1.10 \pm 0.08$ & $0.85 \pm 0.08$ & $0.83 \pm 0.13$ & $(1.19 \pm 0.21)$ & $1.09 \pm 0.58$ \\
\hline Halo & $(0.79 \pm 0.19)$ & $(0.82 \pm 0.11)$ & $0.23 \pm 0.02$ & $(1.70 \pm 0.28)$ & $0.72 \pm 0.06$ & $(2.32 \pm 0.95)$ \\
\hline
\end{tabular}

Notes. The luminosities are corrected for the intrinsic absorption and the absorption in the Milky Way. The luminosities supported by insufficient data are given in brackets. ${ }^{(a)}$ Structural parameters for the young disc are only given for the positive component; see Sect. 2.1 for determining the parameters of the negative component. ${ }^{(b)}$ The luminosities of the young disc are its true luminosities $\left(L=L_{+}+L_{-}\right)$.

considerably different parameters can be fitted to the observational data, yielding somewhat larger deviations.

\section{Results}

In the presented model, the dust disc of M31 has been assumed to consist of a cold component and a warm component. The temperature and intensity distribution maps together with their $1 \sigma$-errors for the warm component are given in Fig. 3. It is seen that the temperature of the warm dust is $56-60 \mathrm{~K}$ in most cases, with a typical uncertainty less than $1 \mathrm{~K}$. The highest temperatures and intensities can be found in the spiral arms, revealing a relation between the warm dust and the star-forming regions. Figure 4 shows the temperature and intensity distribution maps of the cold dust. Temperature of the cold component is usually $15-19 \mathrm{~K}$ with an uncertainty $0.2-1.5 \mathrm{~K}$. In contrast to the warm dust, the temperature of the cold dust rises towards the centre of the galaxy, up to $25 \mathrm{~K}$. This can be explained by a stronger general radiation field resulting from the higher concentration of stars. The intensity distribution of the cold component is much smoother than that of the warm component, matching well with its more diffuse nature. The integral dust temperatures estimated by Gordon et al. (2006), $17 \mathrm{~K}$ for the cold component and $59 \mathrm{~K}$ for the warm component, agree well with the average values derived in our study.

We have used two-dimensional far-IR maps of M 31 for our extinction analysis. For illustration, Fig. 6a shows the Spitzer far-IR measurements, averaged along elliptical isoluminosity contours. In Fig. 6b, the calculated $100 \mu$ m emission distribution is compared to the corresponding observations by IRAS; the low resolution of IRAS prevents from seeing the wavy pattern of the spiral arms. Figure $6 \mathrm{~b}$ also shows the contributions to the $100 \mu \mathrm{m}$ flux by the warm and the cold dust. Except for the very centre of the galaxy, contribution by the warm dust is negligible.

Using Eq. (9), we estimated the ratio of the integral cold dust column density to the warm dust column density to be $(7 \pm 1) \times$ $10^{3}$. Since the space density distribution of dust is proportional to the column density, the given value is a direct estimate of the ratio of the cold dust mass to the warm dust mass. According to Gordon et al. (2006), the mass of the cold dust component exceeds the mass of the warm component by about $10^{4}$ times, close to our estimate. The warm dust gives the highest contribution in the inner bulge region and in the spiral arms, where up to $1 \%$ and up to $0.1 \%$ of the total dust mass can be ascribed to the

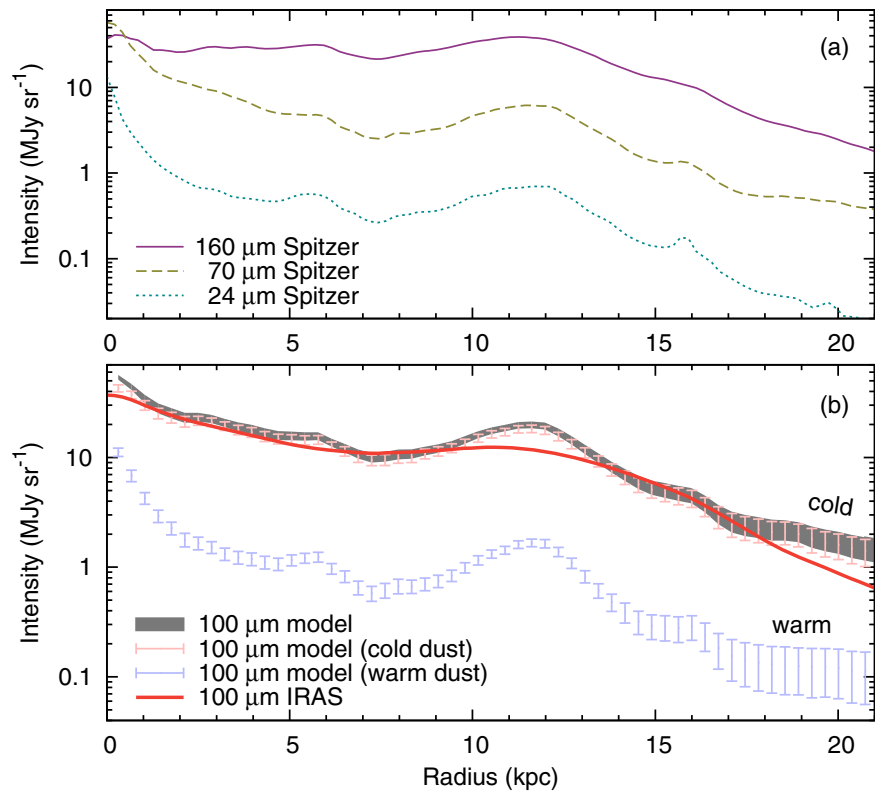

Fig. 6. Far-IR intensity distribution of M31, derived by fitting ellipses to the Spitzer, IRAS, and modelled data. Upper panel - the Spitzer 24, 70 , and $160 \mu \mathrm{m}$ intensity distributions. Lower panel - the modelled intensity at $100 \mu \mathrm{m}$ for the cold and warm components (red and blue errorbars), and their weighted average (filled area); the red solid line represents the IRAS $100 \mu \mathrm{m}$ data.

warm dust, respectively. This demonstrates the insignificance of the warm dust as an absorber of starlight. On the other hand, its contribution has to be estimated and subtracted carefully from far-IR maps to avoid an overestimation of the extinction caused by the cold dust.

The derived maps of the temperature distribution and the $100 \mu \mathrm{m}$ flux of the cold component allowed us to calculate the total optical depth along each line of sight (Fig. 5b). Within the main body of M 31 the total optical depth is $\tau_{\max }=0.8-1.2$ and drops to about 0.3 at the physical limit of the currently available Spitzer far-IR data at about $17 \mathrm{kpc}$. Beyond that, we have assumed its smooth decrease to zero in our model. The map of the total optical depth was used for estimating the intrinsic extinction of M31, but it can also be used for estimating the attenuation of background objects. 
According to the model presented in Sect. 2.2 the number density distribution of the dust disc is represented by an axisymmetric distribution (7). In the $r$-direction, the number density distribution is determined on the basis of the far-IR intensity distribution. The derived number surface density distribution of dust (which is proportional to $\tau_{\max }$ ) for M 31 is given in Fig. $5 \mathrm{c}$. According to the present model, an approximation of the dust disc with a simple exponential law using $N=1$ in Eq. (1) gives $\left(a_{0}\right)_{\text {dust }}=17 \mathrm{kpc}$ as its effective scalelength. The scaleheight of the dust disc is set to be 1.8 times the scaleheight of the stellar disc and is thus $0.24 \mathrm{kpc}$.

The parameters of each stellar component in the best-fit model: the harmonic mean radius $a_{0}$, the axial ratio $q$, the structural parameters $N$, the dimensionless normalising constants $h$ and $k$, the relative size $\kappa$ of the hole in the young disc component, and the absorption-corrected $U B V R I L$-luminosities are given in Table 2, together with the uncertainty estimates. The calculated surface brightness distributions are in good agreement with the observed surface brightness distributions.

Deviations from the observations can be further lowered by increasing the number of components. On the other hand, due to the uncertainties of the surface brightness data and the moderate number of available colours, the component parameters are degenerate and the best-fitted solution is not unique: a change of some parameters of one component can often be compensated with a change of certain parameters of another component. Without including additional observational data, a higher number of components would decrease the uniqueness of the model, probably also decreasing its correspondence to the real galaxy. The aim of the present paper was to map the effects of dust attenuation, thus metallicity determinations in M 31 and chemical evolution model calculations have not been used for laying further constraints on the component parameters. More details on the coupling of the parameters of M 31 can be found in Tenjes et al. (1994). At present, the lack of data leaves the halo to be the most undetermined component, especially in the $U, B, R$, and $L$ colours. Similarly, because of a slight inconsistency of the observational data close to the centre of the galaxy, the $V$ and $R$ colours and the corresponding colour index of the bulge cannot be determined well. The $I$ colour surface brightness distribution is available only along the minor axis. For this reason we cannot clearly distinguish the contributions of the disc and the young disc to the $I$ colour and the corresponding luminosities are rather uncertain.

The axial ratio of the bulge in our best-fit model is $q=1.06 \pm$ 0.03 , referring to a slightly prolate shape of this component. This result seems to be contradicting earlier estimates $q=0.6-0.8$ (de Vaucouleurs 1958; Kent 1989; Tenjes et al. 1994). However, in those studies the intrinsic absorption has not been considered. Extinction is stronger for a given distance along the minor axis than for the same distance along the major axis in the bulge region, causing a decrease of the apparent axial ratio. Without the intrinsic absorption, our model would yield $q=0.8$ as the axial ratio of the bulge, in agreement with previous studies.

To compare the scalelength of the dust disc with that of the stellar disc we fitted a single exponential stellar disc to M31; the model parameters for the bulge and halo components were kept fixes according to the four-component approximation. The single disc has a scalelength $\left(a_{0}\right)_{\text {stars }}=9.2 \mathrm{kpc}$, thus $\left(a_{0}\right)_{\text {dust }}=$ $1.8\left(a_{0}\right)_{\text {stars }}$, which agrees with the seven late-type galaxies studied by Xilouris et al. (1999).

Figure 7 shows the modelled surface brightness distributions along both the major and the minor axes, together with the corresponding observational data. The contribution of the individual components and the intrinsic, dust-corrected surface brightness profiles are also shown. Note that while moving from $U$ to $L$, the young disc component becomes less dominant, because the luminosity of the star-forming ring is dominated by young blue stars. In Figs. 8 and 9, the distributions of the modelled and the observed colour indices are shown.

Figure 10 presents the calculated dust extinction as a function of radius for different filters. To some extent, these distributions are model-dependent, but should be applicable with reasonable precision for correcting any previous or coming observations of the surface brightness distribution of M 31. The largest uncertainties are related to the derivation of the optical depth of the dust disc from the infrared observations. The corresponding errors in Fig. 10 would be of the order of 20 per cent (for the uncertainties of $A_{V}$ see Fig. 11). Dust extinction along the minor axis is higher than along the major axis due to projection effects. The minor axis data represent the closer side of the galaxy, where most of the stars lay behind the dust disc, while along the major axis, the number of stars lying in front and behind the dust disc is equal. This effect is also illustrated by Fig. 7.

The total luminosity of M 31, corrected for the intrinsic absorption is $L_{B}=(3.64 \pm 0.15) \times 10^{10} L_{\odot}$, corresponding to an absolute luminosity of $M_{B}=-20.89 \pm 0.04 \mathrm{mag}$. The dust disc absorbs as much as $20 \%$ (or $0.24 \mathrm{mag}$ ) of the total $B$-flux. Roughly half of the $B$-flux is radiated by the discs $(25 \%$ by the old disc and $30 \%$ by the young disc), about one quarter $(22.5 \%)$ by the bulge, and one quarter $(22.5 \%)$ by the halo. The integrated dustcorrected intrinsic and visible colour indices are given in Table 3.

The ratio of the luminosity of the spheroidal structures (the bulge and the halo) to the total luminosities is 0.45 in $B$ and 0.35 in $V$.

\section{Discussion}

In Fig. 11 we display a comparison of the derived dust extinction profile to some earlier results. Assuming a constant dust to gas ratio, Battaner et al. (1986) have used the H I distribution to estimate visual extinction $A_{V}$ inside M31. Considering the differences in methodology and in the availability of data, their estimate matches well with our extinction profile. Hodge \& Lee (1988) have used the $U-B$ versus $B-V$ colour diagrams to determine reddening inside the disc of the galaxy. If converted into extinction, these values remain somewhat higher than our estimates, especially the $10 \mathrm{kpc}$ measurement, which is off by $0.7 \mathrm{mag}$; however, also the errorbars are large. Xu \& Helou (1996) have estimated the visual optical depth using the IRAS satellite 60 and $100 \mu \mathrm{m}$ maps, an ultra-violet map, H I map, and a model of heating and cooling of dust grains. Converting their optical depth estimates to extinction yields a slightly higher level of extinction than calculated from our model, but Xu \& Helou (1996) have also shown that the calculated optical depth has a large scatter across the galaxy. The most recent analysis of dust inside M 31 was done by Montalto et al. (2009). In their study, the Spitzer data have been processed in a way quite similar to ours. In both studies the main contributor to the far-IR emission is the diffuse dust. Also, the location of the peak attenuation is the same, $10-12 \mathrm{kpc}$ from the centre. According to our calculations, the attenuation decreases towards the outer regions faster than towards the inner regions.

Barmby et al. (2000) and Fan et al. (2008) have measured the mean reddening of globular clusters of $\mathrm{M} 31$ caused by the dust of the galaxy to be $E(B-V)=0.16$ and $E(B-V)=0.28$, respectively. The former value (corresponding to $A_{V}=0.5$ ) agrees with 

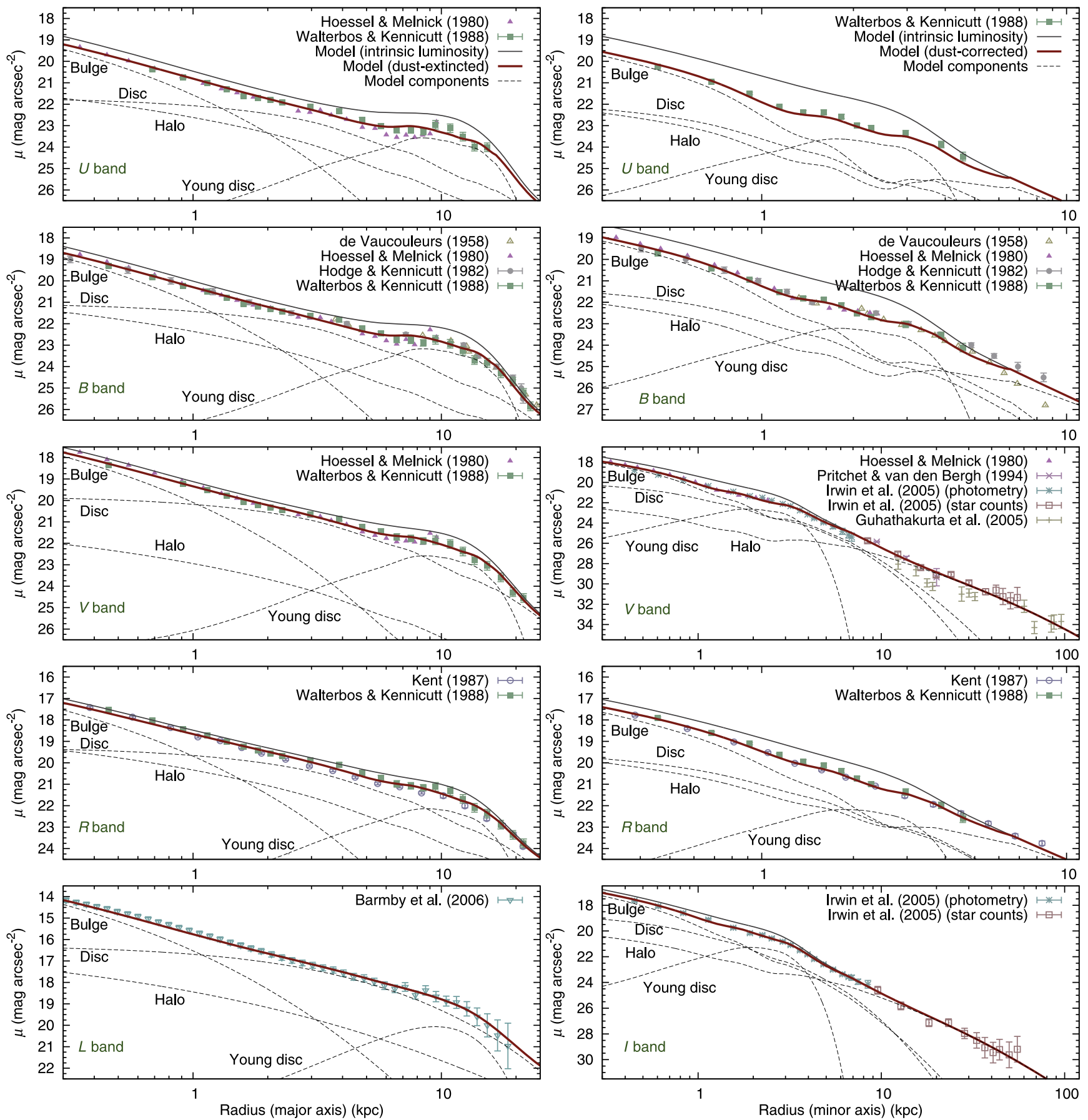

Fig. 7. The observed (data points) and the modelled, dust-extincted (thick red solid lines) surface brightness distributions of M 31 together with the contributions by the individual stellar components (dashed lines). Thin solid lines represent the intrinsic, dust-free surface brightness distributions. Left panels: UBVRL (from top to bottom) surface brightness along the major axis; right panels: UBVRI (from top to bottom) surface brightness along the minor axis.

our estimates, whereas Fan et al. (2008) have derived a somewhat higher extinction $\left(A_{V}=0.87\right)$ than suggested by our model, but once again, the scatter of these reddening estimates is large.

In Fig. 12 we compare our results for the dust temperature and intensity distributions with distributions of molecular gas and young stars. The molecular gas distribution has been measured as the intensity of the CO emission by Nieten et al. (2006); the surface density distribution of a sample of stars with $U-V \leq-0.9$ from Berkhuijsen \& Humphreys (1989) is used as the distribution of young stars. To enable a straightforward comparison, the dust parameters have been measured within a narrow stripe along the major axis, similarly to the measurements of the distributions of gas and stars. No significant correlation is seen between the cold dust temperature and the molecular gas or the young stars (Fig. 12a). The temperature of the cold dust seems to be determined by the general radiation field of the old stars. However, a substantial amount of the emission by the cold diffuse dust can also be powered by non-ionising ultra violet photons coming from young stars, as shown by Popescu et al. (2000) for the case of NGC 891. On the other hand, a correlation exists between the amount (intensity) of the cold dust and the distribution of the molecular gas (Fig. 12c). A difference is 

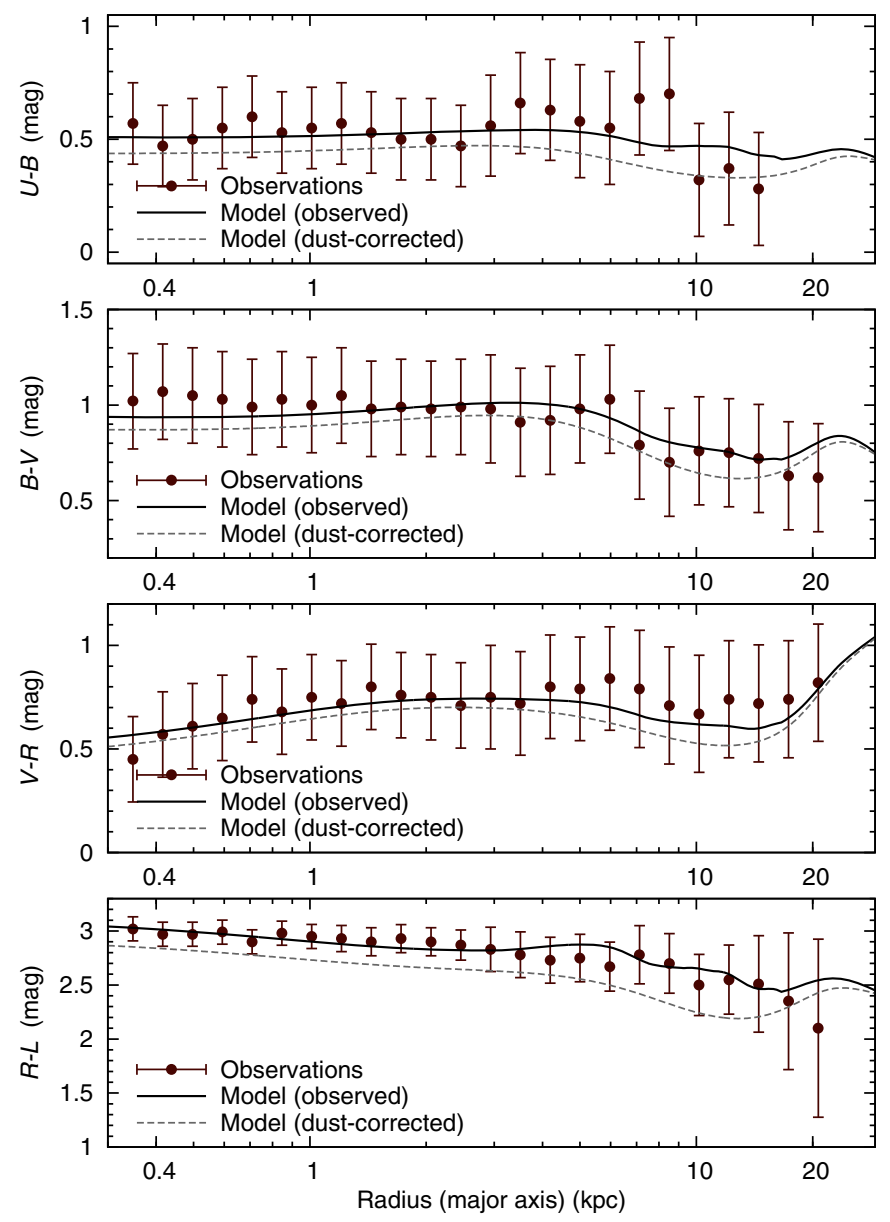

Fig. 8. Distribution of the observed (data points) and modelled (solid lines for the apparent and dashed lines for the dust-corrected) colour indices along the major semi-axis.

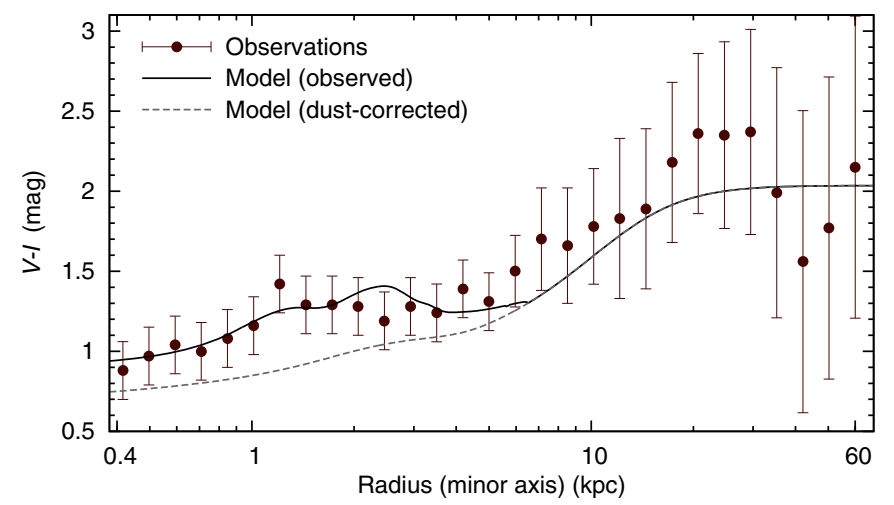

Fig. 9. Distribution of the observed (data points) and modelled (solid lines for observed and dashed lines for dust-corrected) $V-I$ colour index along the minor semi-axis.

only seen at the centre of M 31 where the intensity of the cold dust emission is high while almost no gas is detected. A peak at the centre is also seen in the intensity distribution of the warm dust (Fig. 12d). Correlation between the intensity and temperature distributions of warm dust and young $\mathrm{O}$ stars is moderate only (Figs. 12b and d). The offset can be caused by the tendency to see $\mathrm{CO}$ emission from the trailing edge of the spiral arm, while most of the detectable $24 \mu \mathrm{m}$ emission (radiated by the warm dust) is coming from the leading edge.
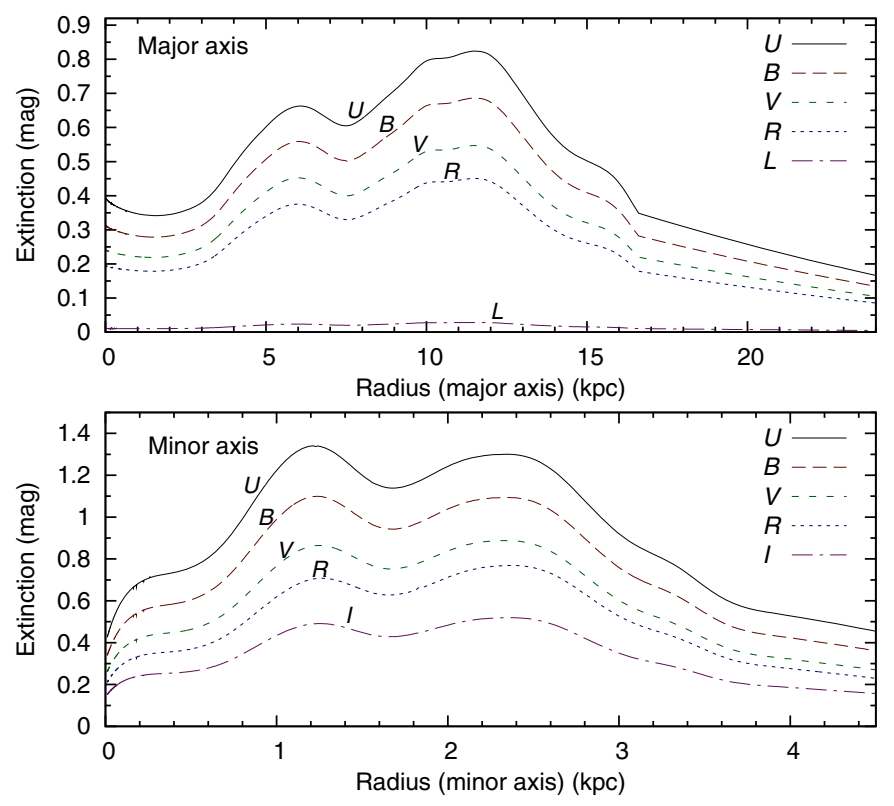

Fig. 10. Dust extinction in $\mathrm{M} 31$ for $U, B, V, R, I$, and $L$ filters, calculated from the model. Upper panel: extinction along the major semiaxis; lower panel: extinction along the minor semi-axis.

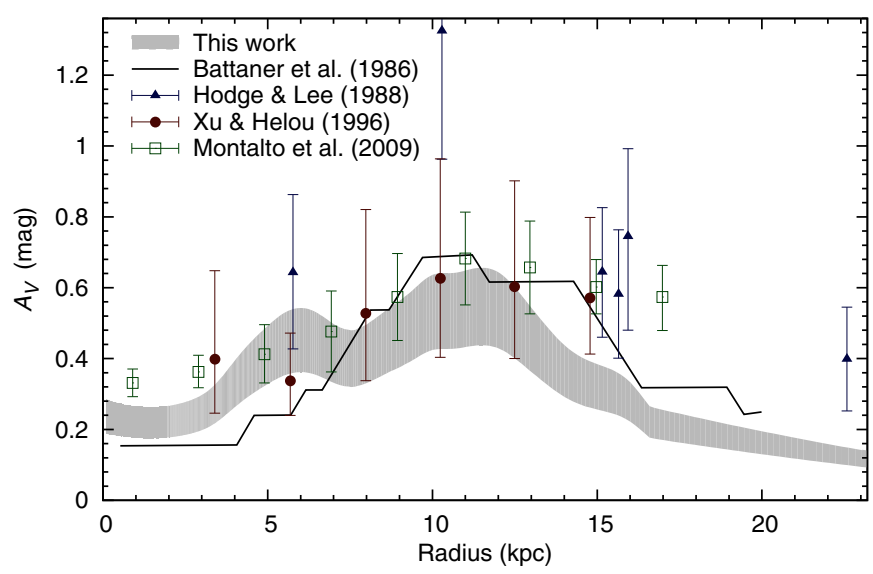

Fig. 11. Calculated dust extinction for the $V$ filter (filled area), compared to earlier estimates by Battaner et al. (1986), Hodge \& Lee (1988), Xu \& Helou (1996), and Montalto et al. (2009).

We have modelled the far-IR spectral energy distribution as a superposition of two modified Planck functions along each line of sight. However, we do not actually know whether the two dust components are physically distinctive or perhaps simply a mathematical description of a single dust disc with higher temperature variations. To provide a description for the case of a single dust component, we have calculated the distribution of the mean intensity-weighted temperature of the dust. In Fig. 13 the mean temperature is given as a function of radius. It is seen that the temperature decreases rather smoothly from $T \sim 32 \mathrm{~K}$ at the centre to $T \sim 20 \mathrm{~K}$ at $R \sim 7 \mathrm{kpc}$ and outwards. For illustration, Fig. 13 also shows the dust temperature distribution for the Scd galaxy NGC 6946, determined using a radiative transfer model by Bianchi et al. (2000); for this plot the radii of NGC 6946 have been multiplied by a factor of 5.9 according to the ratio of the half-light radii of these galaxies. It is seen that although the dust temperature in these galaxies varies within the same range, it decreases more steeply in M 31 indicating a more vivid bulge-disc distinction. 

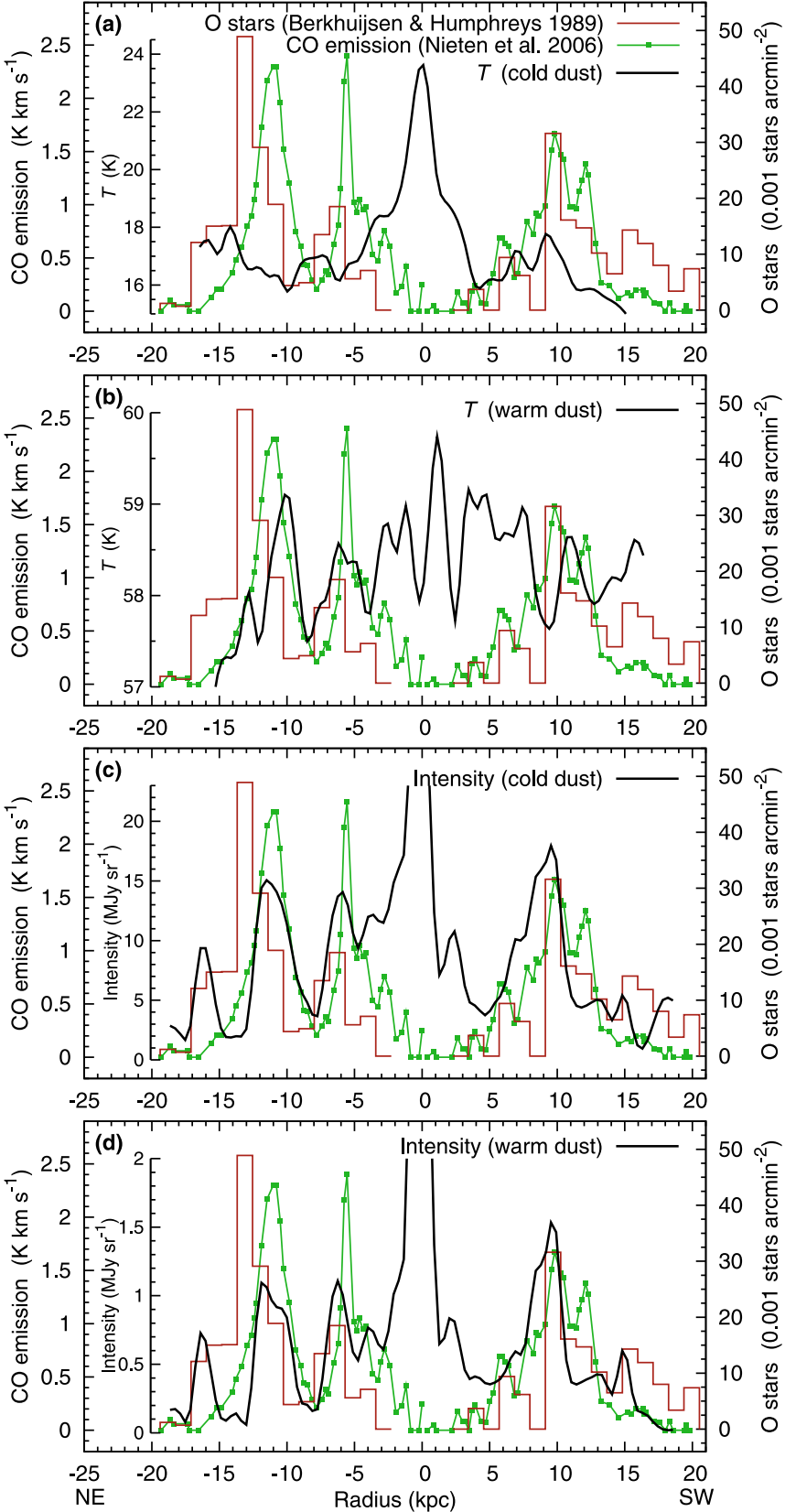

Fig. 12. Distribution of the dust properties derived from present model (black solid lines) together with the distributions of $\mathrm{CO}$ emission (green dotted line, Nieten et al. 2006) and young O stars (red histogram, Berkhuijsen \& Humphreys 1989) along the major axis. a) - cold dust temperature; b) - warm dust temperature; c) - cold dust emission intensity at $100 \mu \mathrm{m}$; d) - warm dust emission intensity at $100 \mu \mathrm{m}$.

Table 3. Intrinsic and visible colour indices of M 31 .

\begin{tabular}{lllll}
\hline \hline M31 & $\begin{array}{l}U-B \\
(\mathrm{mag})\end{array}$ & $\begin{array}{l}B-V \\
(\mathrm{mag})\end{array}$ & $\begin{array}{l}V-R \\
(\mathrm{mag})\end{array}$ & $\begin{array}{l}R-I \\
(\mathrm{mag})\end{array}$ \\
\hline Intrinsic & 0.30 & 0.60 & 0.76 & 0.28 \\
Visible & 0.33 & 0.63 & 0.81 & 0.31 \\
\hline
\end{tabular}

With the help of the presented model, we can also study more general dependencies of dust extinction on the inclination angle, the total optical depth and the dust disc thickness of a galaxy. In Fig. 14 extinction calculations are presented for three dust disc relative thicknesses (the axial ratios $q$ ): $0.005,0.014$, and 0.042 ,

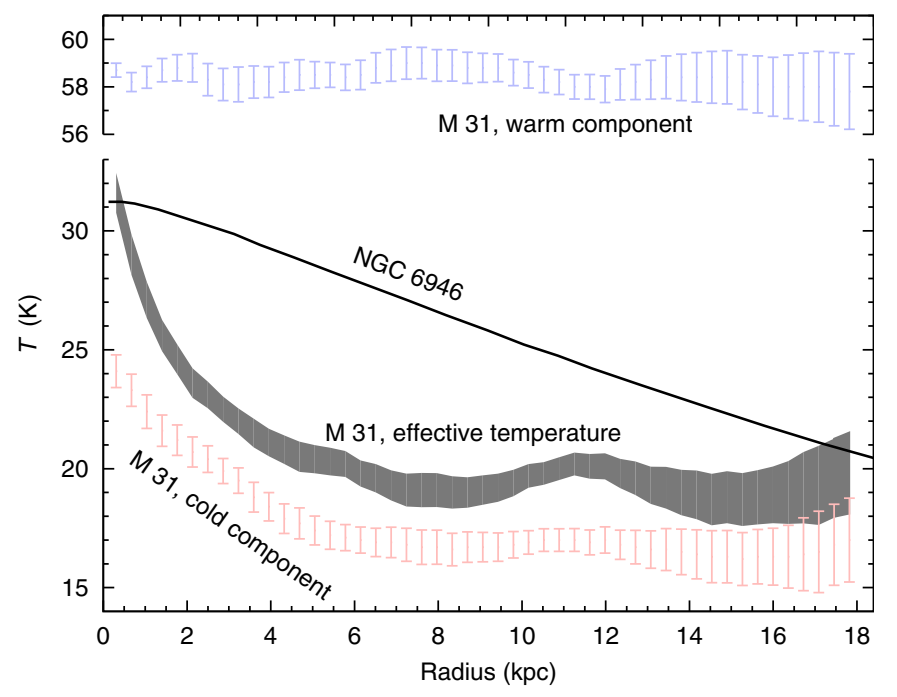

Fig. 13. Temperature distribution of M31, derived by fitting ellipses to the temperature maps. Dark grey area - effective temperature; blue and red errorbars - warm and cold temperature component. The black solid line is temperature derived by Bianchi et al. (2000) for NGC 6946. Temperature distribution of NGC 6946 is scaled to M31 by taking different diameters of these galaxies into account.

designated as "thin", "medium", and "thick", respectively; and for three values of the optical depth $\tau: 0.5 \tau_{\max }, 1.0 \tau_{\max }$, and $2.0 \tau_{\max }$, where $\tau_{\max }$ is the optical depth as derived for M 31 . The other parameters have been set according to the model derived for M31. The vertical line in Fig. 14 refers to the inclination of M31. In the upper panel, the extinction is calculated for the whole galaxy, in the middle panel for a pure bulge component, and in the lower panel for a pure disc component.

In the case of the above-described model of M31, the total extinction would be maximal if the inclination angle of the galaxy were approximately $88^{\circ}$. At lower inclination angles (a more face-on orientation) the total extinction decreases as a result of the decreasing line-of-sight optical depth. For an edgeon galaxy $(1-\cos (i)=1)$, the visible area of the dust disc becomes negligible, and the total extinction is low. It is seen from Fig. 14 that extinction is lower for the disc component than for the bulge and increases more rapidly while moving to higher inclination values. The extinction maximum occurs at higher inclinations for a disc than for a bulge. Similar dependence of dust attenuation on inclination was predicted by Tuffs et al. (2004) and are now confirmed observationally by Driver et al. (2007) using different models and data.

Figure 14 also shows that the extinction maximum shifts slightly towards the lower inclination angles as the optical depth increases; of course, the total extinction increases as well. On the other hand, the total extinction is almost insensitive to the dust disc thickness at low and intermediate inclination angles. This is expected, since the extinction of light emitted outside the dust disc only depends on the optical depth of the dust disc and the dust disc geometry only affects the extinction of light emitted inside the dust disc. In most cases, the line-of-sight thickness of the dust disc is smaller than that of the stellar components and therefore the total extinction does not change when changing the dust disc geometry.

In cases of very high inclination angles, the dust disc thickness becomes much more important, especially if the optical depth is higher as well. However, already for M 31 and galaxies 

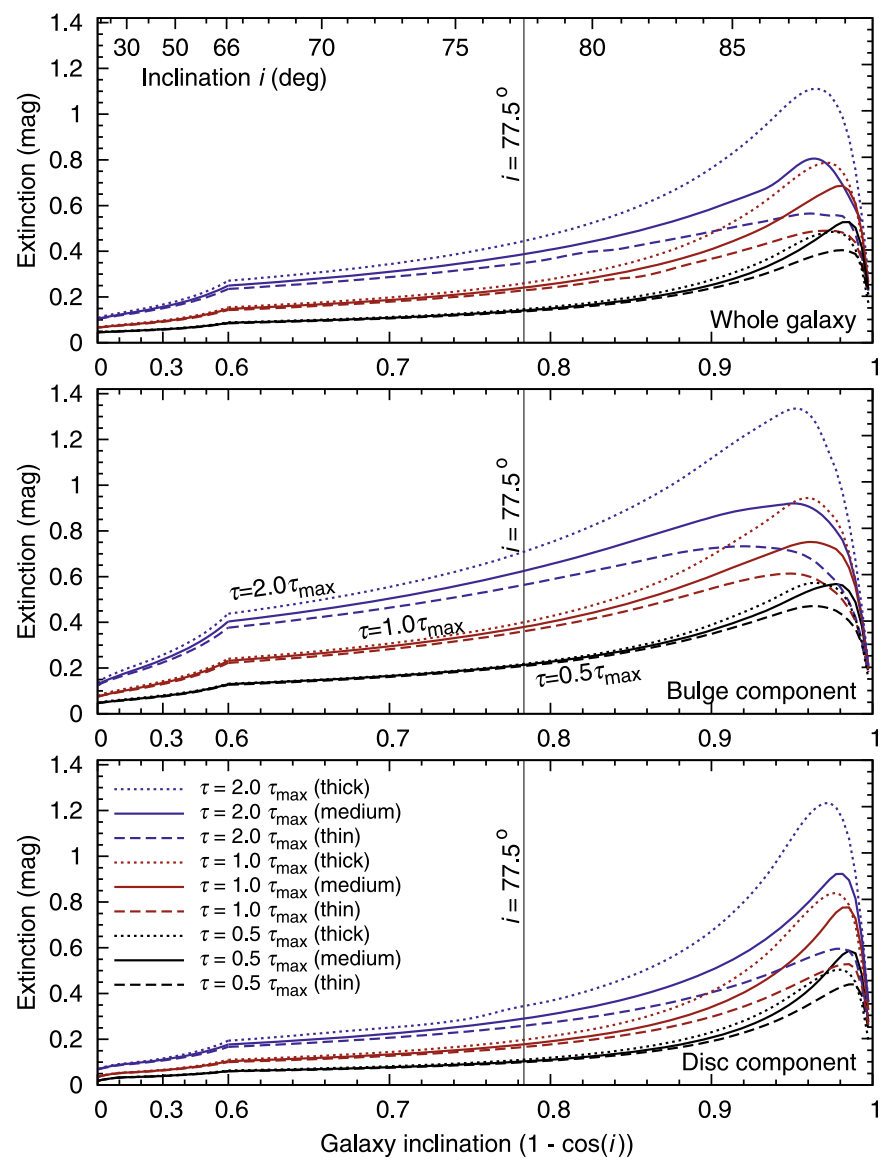

Fig. 14. Integrated dust extinction inside M 31 in $V$-flux as a function of the inclination of the galaxy for a variety of optical depths and dust disc thicknesses. $\tau_{\max }$ is the optical depth used in our model. Upper panel shows the extinction for the whole galaxy, middle panel for a bulge component, and lower panel for a disc-like component. The vertical line represents the inclination angle of M 31 .

at lower inclination angles, the line-of-sight optical depth is the dominant factor.

The dust model used in the present paper involves certain simplifications. Firstly, dust temperatures have not been determined on the basis of a complete radiative transfer model. We have assumed that the distribution of the dust temperature along each line of sight consist of a cold component and of a warm component. The temperature maps have been derived by determining the corresponding four parameters (the temperatures and the intensities of the two components) according to four observational data points for each line of sight. Although mathematically correct, the derived temperatures may be uncertain since the chi-squared approximation is not overdetermined. Secondly, because we do not use a radiative transfer model, the radiation scattering is also ignored. Thirdly, our model does not consider possible small-scale clumpiness of the dust. Although the density behaviour of the dust disc does not follow a simple smooth exponential decrease, having a more complicated structure thanks to the empirically determined correction function $f_{\text {dust }}(r)$ (see Eq. (7)), possible clumps of dust smaller than the resolution of the Spitzer far-IR imaging may be present. The presence of small dust clumps would lead to a lower level of extinction than estimated by our model, since the number of unaffected stars becomes larger.

The vertical thickness of a realistic disc with a spiral pattern should be varying, but the thickness of the old and the young stellar disc is assumed to decrease monotonically in the present model. However, the density distribution of the young stellar disc has been modelled as a ring-like structure with a central density minimum. Since the young disc is thinner than the old disc, their interplay gives a slightly varying total effective thickness for the composite stellar disc. As a result, the ratio of the stellar disc thickness to the dust disc thickness is also variable, being larger in the regions dominated by the old disc and smaller in the other regions. A more detailed study of the structure of the spiral arms remains beyond the scope of the present paper.

The intrinsic luminosity and colour distribution can also be used to derive the visible mass distribution of galaxies. Supplemented with a dynamical model (e.g. Tempel \& Tenjes 2006) and kinematical observations, the visible mass distribution can be used for deriving the distribution of dark matter in galaxies. We will take a closer look at the distribution of visible and dark matter in M31 in a forthcoming paper.

Acknowledgements. We would like to thank the anonymous referee for many constructive suggestions and comments that have helped to improve the manuscript. We thank Dr. J. Pelt for useful hints for improving the fitting process in our photometrical model and for estimating the errors for the parameters of the model. We are indebted to Dr. Karl Gordon, the PI of the Spitzer far-IR observations of M 31. We have also benefited from communications with Dr. Pauline Barmby and Dr. Martin Haas. The Spitzer Science Center and the NASA/IPAC Infrared Science Archive have been of great help in retrieving and processing the infrared data and we are grateful to the team members of these institutions. We acknowledge the financial support from the Estonian Science Foundation grants $7115,7146,7765$ and the project SF0060067s08. All the figures have been made with the gnuplot plotting utility. Many of the timeconsuming computations for this work have been performed at the computer cluster of the University of Tartu.

\section{References}

Barmby, P., Huchra, J. P., Brodie, J. P., et al. 2000, AJ, 119, 727 Barmby, P., Ashby, M. L. N., Bianchi, L., et al. 2006, ApJ, 650, L45 Battaner, E., Beckman, J. E., Mediavilla, E., et al. 1986, A\&A, 161, 70 Beichman, C. A., Neugebauer, G., Habing, H. J., Clegg, P. E., \& Chester, T. J. 1988, Infrared astronomical satellite (IRAS) catalogs and atlases., ed. C. A. Beichman, G. Neugebauer, H. J. Habing, P. E. Clegg, \& T. J. Chester, 1

Berkhuijsen, E. M., \& Humphreys, R. M. 1989, A\&A, 214, 68

Bevington, P. R., \& Robinson, D. K. 2003, Data reduction and error analysis for the physical sciences (Boston: McGraw-Hill)

Bianchi, S. 2007, A\&A, 471, 765

Bianchi, S. 2008, A\&A, 490, 461

Bianchi, S., Davies, J. I., \& Alton, P. B. 2000, A\&A, 359, 65

Brown, T. M., Smith, E., Ferguson, H. C., et al. 2007, ApJ, 658, L95 de Vaucouleurs, G. 1958, ApJ, 128, 465

de Vaucouleurs, G., de Vaucouleurs, A., Corwin, Jr., H. G., et al. 1991, Third Reference Catalogue of Bright Galaxies (Berlin, Heidelberg, New York: Springer-Verlag)

Draine, B. T., \& Li, A. 2007, ApJ, 657, 810

Draine, B. T., Dale, D. A., Bendo, G., et al. 2007, ApJ, 663, 866

Driver, S. P., Popescu, C. C., Tuffs, R. J., et al. 2007, MNRAS, 379, 1022

Durrell, P. R., Harris, W. E., \& Pritchet, C. J. 2001, AJ, 121, 2557

Einasto, J. 1965, Tartu Astr. Obs. Teated, 17

Einasto, J. 1969, Astrofizika, 5, 137

Einasto, J., \& Haud, U. 1989, A\&A, 223, 89

Einasto, J., Tenjes, P., Barabanov, A. V., \& Zasov, A. V. 1980, Ap\&SS, 67, 31

Engelbracht, C. W., Gordon, K. D., Bendo, G. J., et al. 2004, ApJS, 154, 248

Engelbracht, C. W., Blaylock, M., Su, K. Y. L., et al. 2007, PASP, 119, 994

Fan, Z., Ma, J., de Grijs, R., \& Zhou, X. 2008, MNRAS, 385, 1973

Ferguson, A. M. N., Irwin, M. J., Ibata, R. A., Lewis, G. F., \& Tanvir, N. R. 2002, AJ, 124, 1452

Frei, Z., \& Gunn, J. E. 1994, AJ, 108, 1476

González, R. A., Allen, R. J., Dirsch, B., et al. 1998, ApJ, 506, 152

González, R. A., Loinard, L., Allen, R. J., \& Muller, S. 2003, AJ, 125, 1182

Gordon, K. D., Bailin, J., Engelbracht, C. W., et al. 2006, ApJ, 638, L87

Gordon, K. D., Engelbracht, C. W., Fadda, D., et al. 2007, PASP, 119, 1019

Graham, A. W., \& Worley, C. C. 2008, MNRAS, 388, 1708

Guhathakurta, P., Ostheimer, J. C., Gilbert, K. M., et al. 2005

[arXiv: astro-ph/0502366v5], unpublished 
Haas, M., Lemke, D., Stickel, M., et al. 1998, A\&A, 338, L33

Helou, G. 1986, ApJ, 311, L33

Hinz, J. L., Rieke, G. H., Gordon, K. D., et al. 2004, ApJS, 154, 259

Hodge, P. W., \& Kennicutt, R. C. 1982, AJ, 87, 264

Hodge, P. W., \& Lee, M. G. 1988, ApJ, 329, 651

Hoessel, J. G., \& Melnick, J. 1980, A\&A, 84, 317

Holwerda, B. W., González, R. A., Allen, R. J., \& van der Kruit, P. C. 2005, AJ, 129,1396

Holwerda, B. W., Draine, B., Gordon, K. D., et al. 2007, AJ, 134, 2226

Ibata, R., Chapman, S., Ferguson, A. M. N., et al. 2005, ApJ, 634, 287

Ibata, R., Martin, N. F., Irwin, M., et al. 2007, ApJ, 671, 1591

Irwin, M. J., Ferguson, A. M. N., Ibata, R. A., Lewis, G. F., \& Tanvir, N. R. 2005 , ApJ, 628, L105

Kalirai, J. S., Gilbert, K. M., Guhathakurta, P., et al. 2006, ApJ, 648, 389

Kent, S. M. 1987, AJ, 94, 306

Kent, S. M. 1989, AJ, 97,1614

Li, A., \& Draine, B. T. 2001, ApJ, 554, 778

Lonsdale, C. J., \& Helou, G. 1987, ApJ, 314, 513

McConnachie, A. W., Irwin, M. J., Ferguson, A. M. N., et al. 2005, MNRAS, 356, 979

Montalto, M., Seitz, S., Riffeser, A., et al. 2009, A\&A, 507, 283

Muñoz-Mateos, J. C., Gil de Paz, A., Boissier, S., et al. 2009, ApJ, 701, 1965

Nieten, C., Neininger, N., Guélin, M., et al. 2006, A\&A, 453, 459

Padilla, N. D., \& Strauss, M. A. 2008, MNRAS, 388, 1321

Popescu, C. C., \& Tuffs, R. J. 2003, A\&A, 410, L21
Popescu, C. C., Misiriotis, A., Kylafis, N. D., Tuffs, R. J., \& Fischera, J. 2000, A\&A, 362, 138

Pritchet, C. J., \& van den Bergh, S. 1994, AJ, 107, 1730

Sauty, S., Gerin, M., \& Casoli, F. 1998, A\&A, 339, 19

Schlegel, D. J., Finkbeiner, D. P., \& Davis, M. 1998, ApJ, 500, 525

Sérsic, J. L. 1968, Atlas de galaxias australes, Cordoba, Argentina, Observatorio Astronomico

Silva, L., Granato, G. L., Bressan, A., \& Danese, L. 1998, ApJ, 509, 103

Stansberry, J. A., Gordon, K. D., Bhattacharya, B., et al. 2007, PASP, 119, 1038

Tamm, A., \& Tenjes, P. 2006, A\&A, 449, 67

Tempel, E., \& Tenjes, P. 2006, MNRAS, 371, 1269

Tenjes, P., \& Haud, U. 1991, A\&A, 251, 11

Tenjes, P., Haud, U., \& Einasto, J. 1994, A\&A, 286, 753

Tenjes, P., Haud, U., \& Einasto, J. 1998, A\&A, 335, 449

Tuffs, R. J., \& Gabriel, C. 2003, A\&A, 410, 1075

Tuffs, R. J., Popescu, C. C., Völk, H. J., Kylafis, N. D., \& Dopita, M. A. 2004, A\&A, 419, 821

Vlahakis, C., Dunne, L., \& Eales, S. 2005, MNRAS, 364, 1253

Walterbos, R. A. M., \& Kennicutt, Jr., R. C. 1987, A\&AS, 69, 311

Walterbos, R. A. M. \& Kennicutt, Jr., R. C. 1988, A\&A, 198, 61

Weingartner, J. C., \& Draine, B. T. 2001, ApJ, 548, 296

Willmer, C. N. A., Rieke, G. H., LeFloc'h, E., et al. 2009, AJ, 138, 146

Xilouris, E. M., Byun, Y. I., Kylafis, N. D., Paleologou, E. V., \& Papamastorakis, J. 1999, A\&A, 344, 868

Xu, C., \& Helou, G. 1996, ApJ, 456, 163 